\title{
When Differential Pacing Is Not Enough to Assess Mitral Isthmus Block: Importance of the Pacing Threshold
}

\author{
PATRIZIO PASCALE, M.D., ASHOK J. SHAH, M.D., LAURENT ROTEN, M.D., \\ PIERRE JAIS, M.D., and SEBASTIEN KNECHT, M.D., PH.D
}

From the Hôpital Cardiologique du Haut-Lévêque and Université Victor Segalen, Bordeaux II, Bordeaux, France

\begin{abstract}
atrial fibrillation, catheter ablation, left atrial flutter, pulmonary vein isolation
\end{abstract}
\section{Case Presentation}

A 60-year-old man with idiopathic dilated cardiomyopathy was referred for catheter ablation of symptomatic, drugrefractory, persistent atrial fibrillation (AF). The patient was in $\mathrm{AF}$ for a continuous period of 3 years before ablation. A decapolar catheter was positioned inside the coronary sinus (CS) and a $3.5 \mathrm{~mm}$ externally irrigated-tip ablation catheter (Biosense-Webster, Diamond Bar, CA, USA) was used for ablation. After circumferential pulmonary vein isolation, electrogram (EGM)-guided ablation was performed in the left atrium (LA) and inside the CS. Linear ablation at the LA roof and mitral isthmus (MI) was then undertaken during AF. Following electrical cardioversion, LA appendage pacing was performed to evaluate conduction across the MI line. As shown in Figure 1A, a split in the local CS EGMs with a dual pattern of activation is recorded on the decapolar catheter. The persistence of conduction across the MI line is demonstrated by the distal to proximal propagation of the low amplitude and broad EGMs recorded on the CS bipoles. Therefore, further MI ablation was performed during distal $\mathrm{CS}$ pacing and a progressive increase in the MI conduction delay was observed. Using the differential pacing technique, $\mathrm{CS}$ catheter bipoles positioned close to the MI line were sequentially paced to record the time of impulse conduction to the ablation catheter positioned anterior to the MI line. A consistent delay of $166 \mathrm{~ms}$ was recorded on the ablation catheter during pacing at an output of $7 \mathrm{~V}$ and 2-ms pulse width from bipole CS 1-2. Pacing at the same output from bipole CS 3-4 shortened the conduction delay to $150 \mathrm{~ms}$ indicating complete block of conduction from the posterior to the anterior part of the ablation line. As shown in Figure 1B, alternating short and long conduction delays (168 ms and 118

J Cardiovasc Electrophysiol, Vol. 23, pp. 336-338, March 2012.

Patrizio Pascale acknowledges financial support from the Swiss National Science Foundation and the SICPA Foundation.

No disclosures.

Address for correspondence: Patrizio Pascale, M.D., Hôpital Cardiologique du Haut-Lévêque, Avenue de Magellan, 33604 Bordeaux-Pessac, France. Fax: +33-55-765-6509; E-mail: Patrizio.Pascale@ chuv.ch

doi: 10.1111/j.1540-8167.2011.02109.x $\mathrm{ms}$ ) were recorded at the same spot across the MI line during pacing at a higher output of $9 \mathrm{~V}$ and 2-ms pulse width from CS 1-2. The conduction delay remained consistently short (118 ms) when pacing was undertaken with higher output. Short and long conduction delays with high- and low-output pacing, respectively, were similarly observed during CS 3-4 pacing. What is the mechanism underlying the pacing outputdependent block across the MI line?

\section{Commentary}

The dual pattern of CS activation recorded after restoration of sinus rhythm (Fig. 1A) reflects the consequences of ablation inside the CS on connections between the CS and the LA myocardium. On the CS bipoles where a double (split) potential was recorded, the early component consisted of low amplitude and broad EGMs followed by higher amplitude and sharper EGMs. The broader potentials propagate from the lateral CS toward the ostium of the CS up to CS 7-8 whereas the sharper potentials propagate in the opposite direction, i.e., from proximal to distal. The broad EGMs represent "far-field" activity of the adjacent LA myocardium and the sharp EGMs represent the activation of the CS musculature itself. ${ }^{1}$ This pattern, also described as "pseudo" MI block, ${ }^{2}$ provides an evidence of partial disconnection of the mid- and lateral CS (from bipoles CS 1-2 to CS 7-8). The activation of the local CS musculature occurs after the activation of the contiguous LA when the endocardially propagating wavefront reaches the bipoles CS 7-8 and crosses over to the epicardium (CS) at that level.

In the present case, the shorter delay measured across the MI line when pacing at high output close to the line was first hypothesized to result from the "far-field" capture of the LA tissue across the line of block as previously demonstrated. ${ }^{3}$ However, the pacing output-dependent change in the conduction delay was also observed when pacing was undertaken from CS 3-4, a site more distant to the MI line. Moreover, careful examination of CS EGMs on a magnified scale revealed that the activation of the whole CS was modified by changes in pacing output. During high-output CS 1-2 pacing, activation of all recorded CS potentials proceeded uniformly from the distal CS toward the ostium of the CS (Fig. 2A). This pattern therefore suggests that pacing 
Figure 1. A: Recording of a dual pattern of CS activation during pacing from the LA appendage with fluoroscopic image showing the catheter position. B: After further MI line ablation, recordings with RF catheter lying across the MI line of ablation during CS 1-2 pacing. After increasing the pacing output to $9 \mathrm{~V}, 2-\mathrm{ms}$ pulse width, alternating short and long conduction delays (168 $\mathrm{ms}$ and $118 \mathrm{~ms}$ ) are observed. $C S=$ coronary sinus; $R F$ $=$ mapping catheter .

Figure 2. Recordings of the CS activation with amplified scale during CS 1-2 pacing. A: At high output, activation of all recorded CS potentials proceeds from distal to proximal. B: At lower output, a dual pattern of CS activation is observed. The early component, consisting of sharper potentials, demonstrates an activation sequence from the pacing site toward the proximal CS (green arrow). The second broader component demonstrates an activation sequence in the opposite direction, from CS 7-8 toward the distal CS (red arrow). Note that a potential on CS 1-2 is recorded far from the pacing artifact (open arrow).
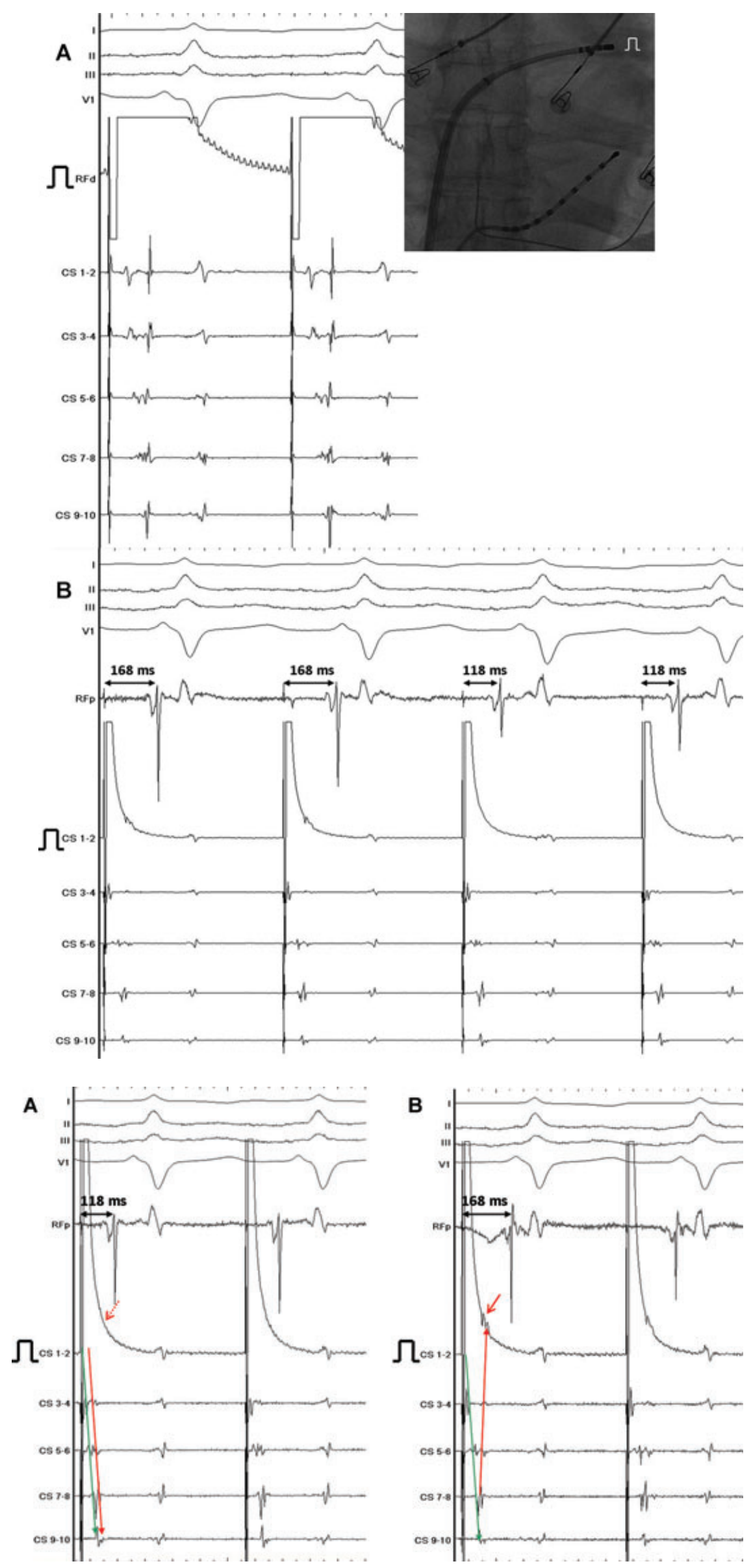


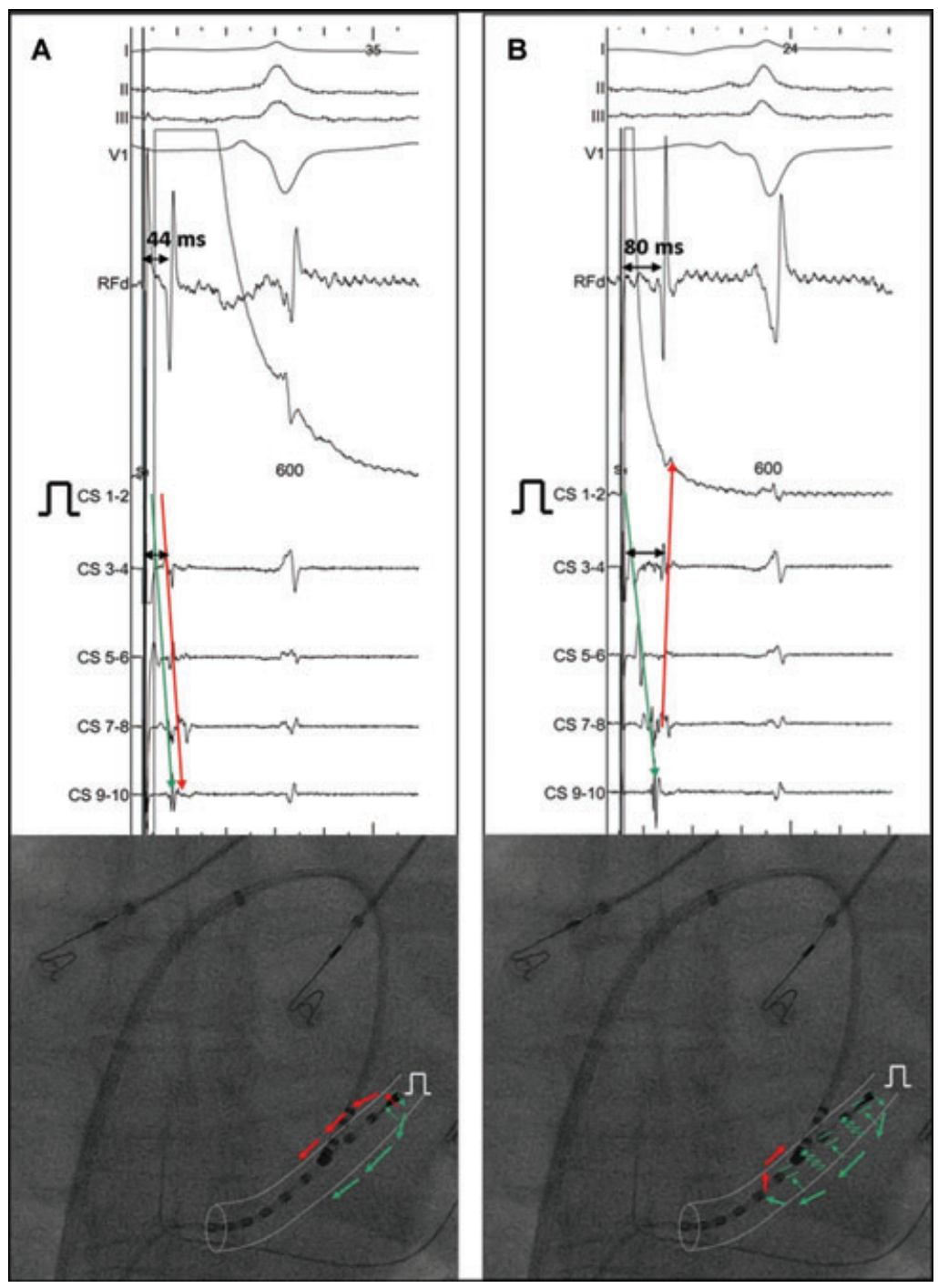

Figure 3. (Top) Intracardiac recordings from the CS and the adjacent LA myocardium during CS 1-2 pacing at high-output (A) and lower-output (B). The ablation catheter is positioned in the LA facing bipole CS 3-4. Note that the potential-recorded endocardially from the LA corresponds temporally to the second component of the double potential recorded on bipole CS 3-4. (Bottom) Fluoroscopic images showing the catheter position with a schematic representation of the distinct activations of the LA myocardium (red arrows) and CS musculature (green arrows).

captured both the CS musculature and the contiguous LA myocardium with a "parallel and isodirectional" activation along the inferior LA. On the other hand, during low-output pacing (Fig. 2B), a dual pattern of CS activation was again observed. The early component, consisting of sharper EGMs, demonstrated an activation sequence from the pacing site (CS 1-2) toward the ostium of the CS (green arrow). The second broader component, presumably representing the "far-field" LA myocardium, demonstrates an activation sequence in the opposite direction, from CS 7-8 toward the distal CS (red arrow). It appears therefore that the pacing stimulus captured the CS musculature but not the contiguous LA. Because of the previously demonstrated disconnection of the CS from contiguous LA (spanning from CS 1-2 to CS 7-8), the activation wavefront proceeds along the CS musculature up to CS 7-8. At that level, it traverses over the residual LA/CS connection to reach the endocardium and activates the LA myocardium in the direction from CS 7-8 to CS 1-2. The hypothesis that the second component of the split EGM recorded on the CS bipole 3-4 represents the "far-field" LA was validated by positioning the ablation catheter endocardially facing the bipole CS 3-4. As shown in Figure 3, the potential recorded on the ablation catheter corresponds temporally to the second potential on CS 3-4 during high- and lower-output pacing (Fig. 3A,B, respectively).

This case demonstrates that, due to previous ablation, capture of the CS musculature may occur without capture of the adjacent LA myocardium. It can mislead the interpretation of differential pacing during evaluation of MI block because the ensuing activation wavefront "detour" during CS pacing at low output can mimic conduction block across the MI. Concomitant capture of both CS musculature and adjacent LA should therefore always be ascertained by careful inspection of all recorded CS potentials.

\section{References}

1. Antz M, Otomo K, Arruda M, Scherlag BJ, Pitha J, Tondo C, Lazzara R, Jackman WM: Electrical connections between the right atrium and the left atrium via the musculature of the coronary sinus. Circulation 1998; $98: 1790-1795$.

2. Takatsuki S, Extramania F, Hayashi M, Leenhardt A: Conduction through the lateral mitral isthmus: Block or pseudoblock? J Cardiovasc Electrophysiol 2008;19:14-18.

3. Miyazaki S, Liu X, Shah A, Haïssaguerre M, Hocini M: Differential pacing for confirming mitral isthmus block: Is the mitral isthmus completely blocked? Heart Rhythm 2011;8:145-147. 